\title{
含冠醚双亲共聚物纳米聚集体的制备及性能
}

\author{
张㻦健 ${ }^{a, b} \quad$ 谢涁 ${ }^{b}$ 姜涛*, $a$ \\ $\left({ }^{a}\right.$ 天津科技大学 材料科学与化学工程学院 天津 300457) \\ ( ${ }^{b}$ 中国石油石油化工研究院 合成树脂加工应用研究室 北京 100195)
}

\begin{abstract}
摘要 模拟 DNA 化学结构, 设计并合成了双亲共聚物聚(2,2'-(1,10-二氮杂-[18]冠-6-1,10-二基)二乙基 5-((腺嘌呤-9-基) 甲基)间苯二甲酸酯) (PDCAI), 利用扫描电镜(SEM)观测了其在水溶液中的自组织形态, 采用红外光谱法(FT-IR)研究了 其与底物胸腺嘧啶(thymine)的氢键识别, 并以变温红外进一步证实和考察了氢键的形成及断裂. 同时, 尝试了利用 $\mathrm{K}^{+}$ 对 PDCAI 的自组织形态和氢键识别进行了调控, 结果表明: 在水溶液中 PDCAI 自发聚集成条带状聚集体, 利用 $\mathrm{K}^{+}$调 控可使其聚集形态转变为棒状、纳米管状或螺旋棒状; 在水溶液中底物 thymine 的 $\mathrm{C}_{2}=\mathrm{O}$ 与 PDCAI 进行了氢键识别, 而 通过 $\mathrm{K}^{+}$调控, 氢键识别基变为 thymine 的 $\mathrm{C}_{4}=\mathrm{O}$, 说明 PDCAI 聚集形态的转变导致 thymine 在与其识别过程中进行识 别构象的重组织. PDCAI 的研制对研究揭示聚合物自发形成螺旋的分子特征、制备螺旋型聚合物、研制新型药物载体 及功能调控具有参考意义.
\end{abstract}

关键词＼cjkstart药物载体; 聚集体; 氢键; 离子调控

\section{Preparation and Properties of Crown Ethers Containing Amphiphilic Copolymer Nano-aggregates}

\author{
Zhang, Yu-Jian ${ }^{a, b} \quad \mathrm{Xie}, \mathrm{Bin}^{b} \quad$ Jiang, Tao ${ }^{*, a}$ \\ ( ${ }^{a}$ Tianjin University of Science and Technology, Materials Science and Chemical Engineering Institute, \\ Tianjin 300457, China)
}

$\left({ }^{b}\right.$ Petrochemical Research Institute of PetroChina, Synthetic Resins and Processing Applications Lab, Beijing 100195, China)

\begin{abstract}
In this paper, an amphiphilic copolymer poly(2,2'-(1,10-diaza-[18]crown-6-1,10-diyl)diethyl 5-((adenin-9-yl)methyl)isophthalate) (PDCAI) was designed and synthesized by simulating the chemical structure of DNA. We observed its self-organized morphology in the aqueous solution and in potassium solution with scanning electron microscopy (SEM), amphiphilic copolymer PDCAI spontaneously aggregated into strip aggregates in aqueous solution, and which could change into a rod, nanotube or helical rod aggregates in $\mathrm{KNO}_{3}$ solution. In addition, the molecular recognition between copolymer PDCAI and thymine substrate has been studied via FT-IR, and it is found that $\mathrm{C}_{2}=\mathrm{O}$ of thymine had recognized with PDCAI through complementary nucleobases in aqueous solution, the $\mathrm{C}_{2}=\mathrm{O}$ stretching band of thymine at $1737 \mathrm{~cm}^{-1}$ shifted to 1710 $\mathrm{cm}^{-1}$ after recognition, however, the band of the $\mathrm{C}_{4}=\mathrm{O}$ of thymine did not change at $1677 \mathrm{~cm}^{-1}$. Meanwhile we attempted to regulate the molecular recognition of copolymer PDCAI with thymine substrate with $\mathrm{K}^{+}$, we surprisingly found that hydrogen bonding occurs on $\mathrm{C}_{4}=\mathrm{O}$ of thymine when it recognized with PDCAI in $\mathrm{KNO}_{3}$ solution, the $\mathrm{C}_{4}=\mathrm{O}$ stretching band of thymine at $1677 \mathrm{~cm}^{-1}$ shifted to $1671 \mathrm{~cm}^{-1}$, however, the band of the $\mathrm{C}_{2}=\mathrm{O}$ of thymine did not change at $1737 \mathrm{~cm}^{-1}$ after recognition. It proves that the recognizing conformation of thymine re-organized during identification process due to the transition of aggregation form of PDCAI. And we further confirmed and studied the hydrogen bond formation and fracture process by variable temperature FT-IR, which formed at room temperature gradually broke while temperature rising from $25{ }^{\circ} \mathrm{C}$ up to $115{ }^{\circ} \mathrm{C}$, when temperature was above $115{ }^{\circ} \mathrm{C}$, hydrogen bonds broke completely, thymine and PDCAI return to their pre-recognition state. The formations of hydrogen bonds between adenine in the polymer and thymine substrate in nanospheres could enhance their interaction and loading capacity. The results have reference value for research of molecular characteristics of polymer which spontaneously formed spiral, preparation of helical polymer, and nucleic acid imitation drug carriers and its function regulation.
\end{abstract}

Keywords drug carriers; aggregates; hydrogen bond; ion regulation

1 引言

亲脂疏水作用和氢键作用为生物体内的两种重要
的弱相互作用，对生物高分子的形成及其生命功能的维 系至关重要，如: DNA 双螺旋的形成、复制以及生物膜 的构建 ${ }^{[1 \sim 4]}$. 在过去的几十年中，这两种作用被普遍应

\footnotetext{
*E-mail: jiangtao@tust.edu.cn

Received June 7, 2016; published September 5, 2016.

Key Projects of Application Foundation and Frontier Technology Research Program of Tianjin Municipality (16JCZDJC31600).

项目受天津市应用基础与前沿技术研究计划重点项目(16JCZDJC31600)资助.
} 
用于聚合物囊泡、药物载体和靶向药物制剂的研制 ${ }^{[5 \sim 8]}$, 通常，在水环境中由于水的竞争，通过氢键作用进行的 分子识别无法进行 ${ }^{[9]}$, 因此, 人们设计了多种双亲性聚 合物(受体), 其依赖亲脂疏水作用驱动自组织形成超分 子疏水区域(纳米聚集体), 可为药物(底物)的包埋和识 别提供疏水的微环境, 排除了环境水的竞争性干扰 ${ }^{[10]}$, 同时, 通过氢键识别又可进一步提高受体对底物的负载 量, 研究这一识别过程(特别是聚集体内部的氢键识别) 的有效手段之一为傅立叶红外光谱法(FT-IR $)^{[11,12]}$.

聚合物的双亲性是其在水溶液中自发聚集的前提, 从化学结构角度分析, DNA 为双亲链状高分子, 聚合链 中的疏水基为核苷, 亲水基为磷脂, 其双螺旋的形成与 自身双亲性及互补链间的氢键作用密切相关 ${ }^{[13]}$, 另外, 以 DNA 为靶在抗癌药物的设计方面具有特殊意义 ${ }^{[14]}$. 冠醚兼备良好的水溶性和脂溶性, 同时具有良好的络合 金属离子的能力 ${ }^{[15 ~ 18]}$, 本文中我们仿照 DNA 化学结构, 以亲水性的冠醚模拟磷脂, 以疏水性的腺嘌呤代间苯二 甲酸酯模拟核苷, 设计合成了生物相容可降解双亲共聚 物聚 $\left(2,2^{\prime}-(1,10\right.$-二氮杂 -[18] 冠 -6-1,10-二基)二乙基 5-((腺嘌呤-9-基)甲基)间苯二甲酸酯) (PDCAI), 扫描电 镜(SEM)观测了其在水溶液中的自组织形态, 采用红外 光谱法研究了其与底物胸腺嘧啶(thymine)的氢键识别, 并以变温红外证实和考察了氢键的形成及断裂. 另外尝 试了利用 $\mathrm{K}^{+}$对共聚物 PDCAI 的聚集形态及其与 thymine 的氢键识别进行了调控.

\section{2 结果与讨论}

\section{1 共聚物 PDCAI 的聚集形态}

共聚物 PDCAI 在水溶液中的聚集形态见图 1, 由图 1 可看出其自发聚集成了宽约 40 $50 \mathrm{~nm}$ 的条带状聚集 体, 可能由于在纯水溶液中冠醚环以近似于柔链状形式 存在，因而共聚物 PDCAI 呈现柔性聚集形态 ${ }^{[16]}$.

另外, 鉴于冠醚对金属离子良好的络合能力, 选择 与氮杂-18-冠-6 环大小匹配的 $\mathrm{K}^{+}$作为金属络合物 ${ }^{[16]}$, 将共聚物 PDCAI 溶于 $\mathrm{KNO}_{3}$ 水溶液中(冠醚官能团与钾 离子摩尔比 1:1) 并观测其聚集形态(见图 2), 可看出, PDCAI 的聚集形态转变为棒状或纳米管状, 部分呈现 了螺旋棒状, 可能因为 $\mathrm{K}^{+}$与冠醚环的络合使其由近

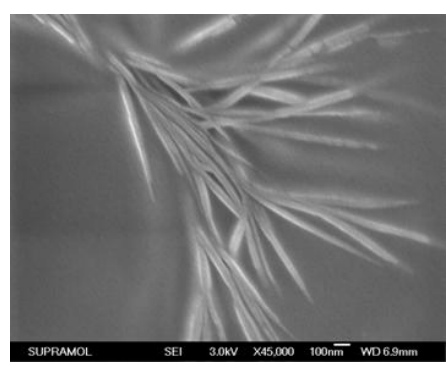

图 1 水溶液 $\left(25{ }^{\circ} \mathrm{C}\right)$ 中共聚物 PDCAI 的扫描电镜照

Figure 1 The SEM image of copolymer PDCAI in water at $25{ }^{\circ} \mathrm{C}$

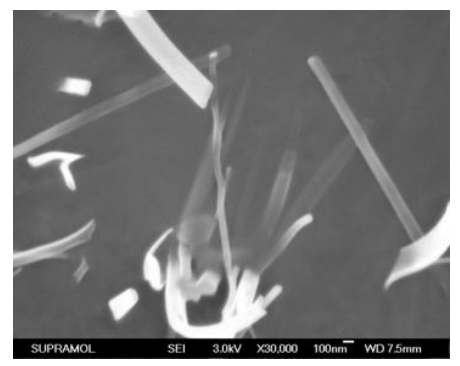

图 2 硝酸钾水溶液 $\left(25{ }^{\circ} \mathrm{C}\right)$ 中共聚物 PDCAI 的扫描电镜照

Figure 2 The SEM image of copolymer PDCAI in potassium solution at $25{ }^{\circ} \mathrm{C}$

似链状转变为刚性环状 (类似于苯环 $)^{[16]}$, 从而导致了共 聚物 PDCAI 聚集形态的转变.

\section{$2.2 \mathrm{PDCAI}$ 与胸腺嘧啶的分子识别}

制备 PDCAI、PDCAI/thymine 纳米聚集体，冻干后 与 $\mathrm{KBr}$ 混合压片待测红外光谱.

\subsection{1 水溶液中 PDCAI 与胸腺嘧啶的分子识别}

图 3 和图 4 是共聚物 PDCAI 纳米聚集体、 $\mathrm{PDCAI} /$ thymine 纳米聚集体(水溶液中制备)、底物 thymine 分别在 $1800 \sim 1520 \mathrm{~cm}^{-1}$ 和 $3750 \sim 2600 \mathrm{~cm}^{-1}$ 范 围内的常温红外谱图.

如图 3 所示, 在 PDCAI 谱线中 $1718 \mathrm{~cm}^{-1}$ 为共聚物 主链上酯羰基的伸缩振动峰, $1616 \mathrm{~cm}^{-1}$ 为共聚物中腺 嘌呤上伯胺 N-H 变形振动峰 ${ }^{[19]}$. 在 thymine 谱线中 1737 $\mathrm{cm}^{-1}$ 和 $1677 \mathrm{~cm}^{-1}$ 分别为 thymine 环上 $\mathrm{C}_{2}=\mathrm{O}$ 和 $\mathrm{C}_{4}=\mathrm{O}$ 伸缩振动峰.

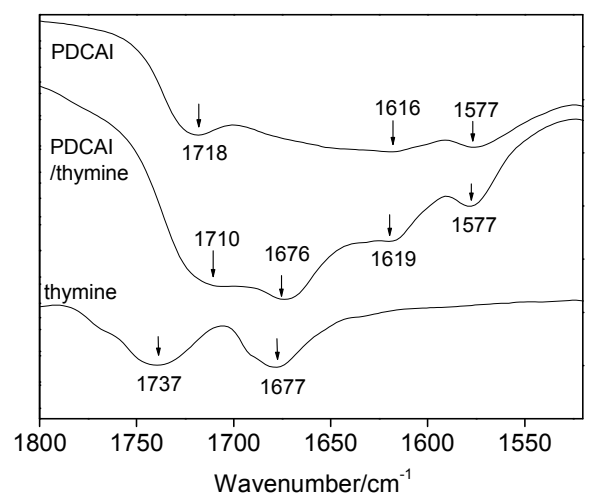

图 3 共聚物 PDCAI 纳米聚集体、PDCAI/thymine 纳米聚集体、 thymine 在 $1800 \sim 1520 \mathrm{~cm}^{-1}$ 范围内的红外谱图

Figure 3 FT-IR spectra of PDCAI polymer nano-aggregates, $\mathrm{PDCAI} /$ thymine nano-aggregates and thymine in $1800 \sim 1520 \mathrm{~cm}^{-1}$

对比可知, 在 PDCAI/thymine 谱线中, thymine 环上 $\mathrm{C}_{2}=\mathrm{O}$ 伸缩振动峰由 $1737 \mathrm{~cm}^{-1}$ 红移至 $1710 \mathrm{~cm}^{-1}$ 处(与 共聚物主链上酯羰基峰重合), 而 thymine 环上 $\mathrm{C}_{4}=\mathrm{O}$ 伸 缩振动峰 $1677 \mathrm{~cm}^{-1}$ 基本没有变化, 说明底物 thymine 环上 $\mathrm{C}_{2}=\mathrm{O}$ 与 PDCAI 中腺嘌呤上 $-\mathrm{NH}_{2}$ 间发生氢键作用.

如图 4 所示, 在 PDCAI 谱线中 $3314 \mathrm{~cm}^{-1}$ 为 PDCAI 
中腺嘌呤上- $\mathrm{NH}_{2}$ 伸缩振动峰 ${ }^{[20]}$. 在 thymine 谱线中 3201 $\mathrm{cm}^{-1}$ 为伯酰胺氢键缔合伸缩振动峰.

在 PDCAI/thymine 谱线中, 伸缩振动峰 $3314 \mathrm{~cm}^{-1}$ 蓝移至 $3399 \mathrm{~cm}^{-1}$ 处, 说明 PDCAI 中腺嘌呤上- $\mathrm{NH}_{2}$ 与 thymine 上羰基发生了氢键作用 ${ }^{[21]}$, 并出现了 3205 、 $3106 、 3048 \mathrm{~cm}^{-1}$ 三处新的氢键缔合伸缩振动峰.

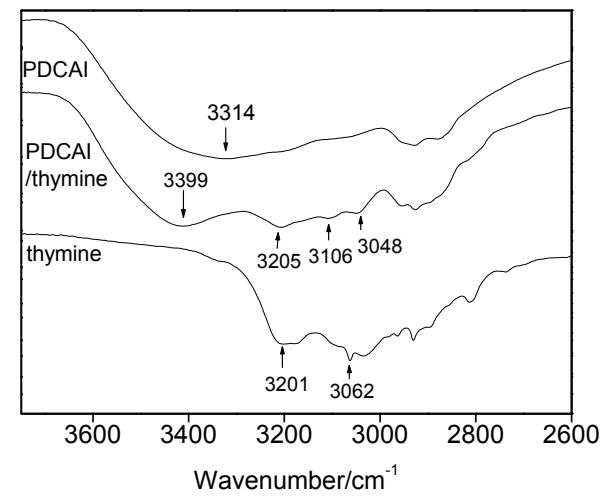

图 4 共聚物 PDCAI 纳米聚集体、 PDCAI/thymine 纳米聚集体、 thymine 在 $3750 \sim 2600 \mathrm{~cm}^{-1}$ 范围内的红外谱图

Figure 4 FT-IR spectra of PDCAI polymer nano-aggregates, $\mathrm{PDCAI} /$ thymine nano-aggregates and thymine in $3750 \sim 2600 \mathrm{~cm}^{-1}$

为了验证 $\mathrm{PDCAI} /$ thymine 纳米聚集体(水溶液中制 备)内部氢键作用的发生, 将其进行了变温红外测试(图 5 和图 6). 如图 5 所示, 随着温度的升高, thymine 环上 $\mathrm{C}_{4}=\mathrm{O}$ 峰 $1676 \mathrm{~cm}^{-1}$ 基本无变化, 而识别后的新峰 1710 $\mathrm{cm}^{-1}$ 逐渐消失, 当温度 $\geqslant 115{ }^{\circ} \mathrm{C}$ 时, 出现 $1735 、 1716$ 、 $1697 、 1681 \mathrm{~cm}^{-1}$ 四个峰, 对比可知, $1716 \mathrm{~cm}^{-1}$ 为识别前 PDCAI 主链上酯羰基伸缩振动峰, $1735 \mathrm{~cm}^{-1}$ 为识别前 thymine 环上 $\mathrm{C}_{2}=\mathrm{O}$ 伸缩振动峰, $1697 \mathrm{~cm}^{-1}$ 为 thymine 环 $\mathrm{C}=\mathrm{C}$ 和 $\mathrm{C}_{4}=\mathrm{O}$ 联合振动峰 ${ }^{[22]}, 1681 \mathrm{~cm}^{-1}$ 腺嘌呤上 $-\mathrm{NH}_{2}$ 变形振动峰.

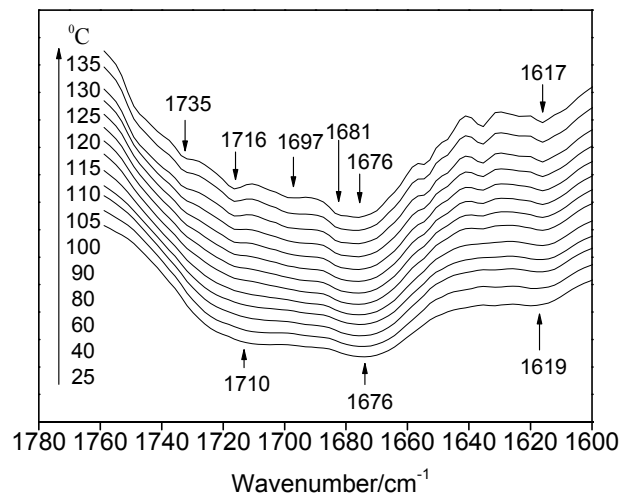

图 $525 \sim 135{ }^{\circ} \mathrm{C}$ 范围内 PDCAI/thymine 纳米聚集体在 $1800 \sim 1520$ $\mathrm{cm}^{-1}$ 范围内的变温红外谱图

Figure 5 Variable temperature FT-IR spectra of PDCAI/thymine nano-aggregates in $1800 \sim 1520 \mathrm{~cm}^{-1}$ at $25 \sim 135{ }^{\circ} \mathrm{C}$

如图 6 所示, 随温度升高识别后出现的新峰 3399、
3205、3106、3048 $\mathrm{cm}^{-1}$ 逐渐消失, 最终出现了 3199 和 $3063 \mathrm{~cm}^{-1}$ 两个透射峰，与识别前 thymine 谱线中两个峰 位基本一致(参考图 4). 变温红外测试结果证明常温下 底物 thymine 与 PDCAI 间形成的氢键高温时逐渐断裂, 当温度 $\geqslant 115{ }^{\circ} \mathrm{C}$ 时氢键断裂完全, PDCAI 和 thymine 各 自回归到识别前的状态.

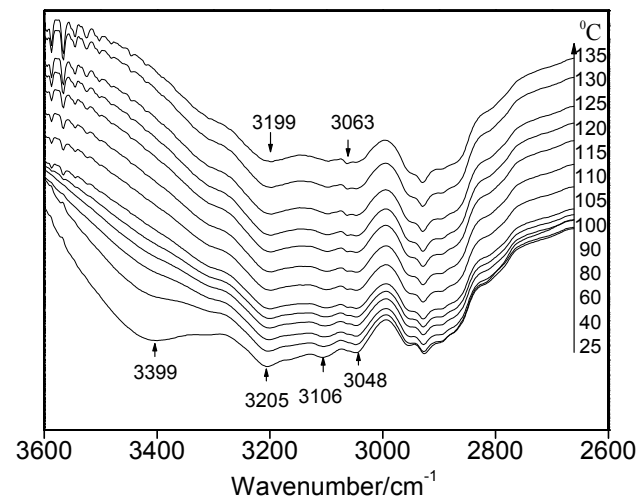

图 $625 \sim 135{ }^{\circ} \mathrm{C}$ 范围内 $\mathrm{PDCAI} /$ thymine 纳米聚集体在 $3750 \sim 2600$ $\mathrm{cm}^{-1}$ 范围内的变温红外谱图

Figure 6 Variable temperature FT-IR spectra of PDCAI/thymine nano-aggregates in $3750 \sim 2600 \mathrm{~cm}^{-1}$ at $25 \sim 135{ }^{\circ} \mathrm{C}$

\subsection{2 $\mathrm{KNO}_{3}$ 水溶液中 PDCAI 与胸腺嘧啶的分子识别}

图 7 和图 8 是共聚物 PDCAI 纳米聚集体、 $\mathrm{PDCAI} /$ thymine 纳米聚集体 $\left(\mathrm{KNO}_{3}\right.$ 水溶液中制备)、底物 thymine 分别在 $1800 \sim 1520 \mathrm{~cm}^{-1}$ 和 $3750 \sim 2600 \mathrm{~cm}^{-1}$ 范 围内的常温红外谱图.

如图 7 所示, 在 PDCAI/thymine 谱线中 $1739 \mathrm{~cm}^{-1}$ 和 $1720 \mathrm{~cm}^{-1}$ 分别为 thymine 环上 $\mathrm{C}_{2}=\mathrm{O}$ 伸缩振动峰和 PDCAI 主链酯羰基伸缩振动峰, 这两个峰位与识别前 基本无变化, 但识别后 thymine 环上 $\mathrm{C}_{4}=\mathrm{O}$ 伸缩振动峰 由 $1677 \mathrm{~cm}^{-1}$ 位移至 $1671 \mathrm{~cm}^{-1}$ 处, 说明其与 PDCAI 中 腺嘌呤上 $-\mathrm{NH}_{2}$ 基间发生了氢键作用.

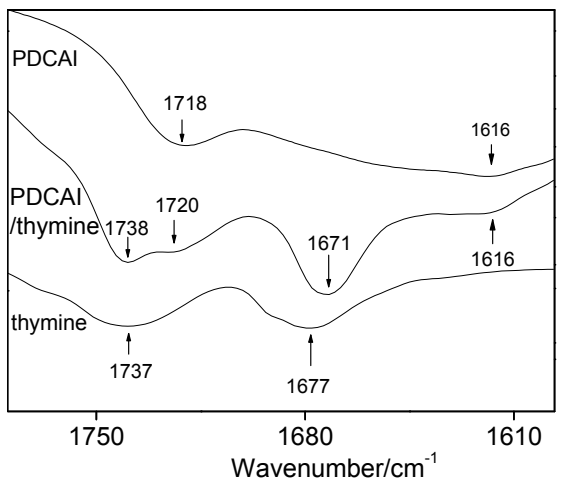

图 7 硝酸钾水溶液中制备的共聚物 PDCAI 纳米聚集体、 PDCAI/thymine 纳米聚集体、 thymine 在 $1780 \sim 1595 \mathrm{~cm}^{-1}$ 范围内的红 外谱图

Figure 7 FT-IR spectra of PDCAI polymer nano-aggregates, PDCAI/thymine nano-aggregates and thymine in $1780 \sim 1595 \mathrm{~cm}^{-1}$ prepared from potassium nitrate aqueous solution 
如图 8 所示, PDCAI/thymine 谱线中 PDCAI 中腺嘌 呤上 $-\mathrm{NH}_{2}$ 伸缩振动峰由识别前的 $3314 \mathrm{~cm}^{-1}$ 蓝移至 $3398 \mathrm{~cm}^{-1}$ 处, thymine 中伯酰胺氢键缔合伸缩振动峰 $3201 \mathrm{~cm}^{-1}$ 识别后蓝移至 $3210 \mathrm{~cm}^{-1}$ 处.

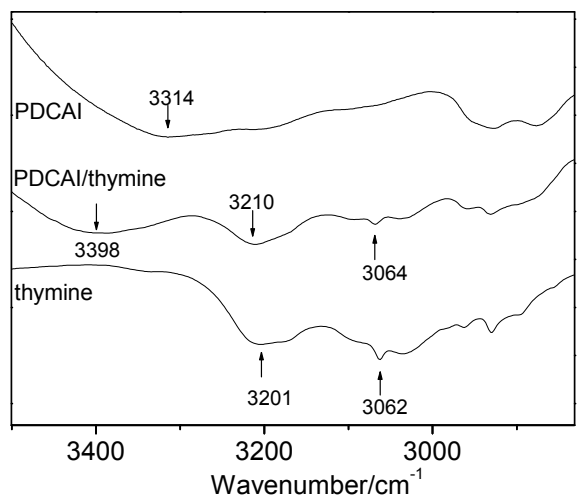

图 8 硝酸钾水溶液中制备的共聚物 PDCAI 纳米聚集体、 PDCAI/thymine 纳米聚集体、thymine 在 $3500 \sim 2800 \mathrm{~cm}^{-1}$ 范围内的红 外谱图

Figure 8 FT-IR spectra of PDCAI polymer nano-aggregates, $\mathrm{PDCAI} /$ thymine nano-aggregates and thymine in $3500 \sim 2800 \mathrm{~cm}^{-1}$ prepared from potassium nitrate aqueous solution

同样为了验证 $\mathrm{PDCAI} /$ thymine 纳米聚集体 $\left(\mathrm{KNO}_{3}\right.$ 水溶液中制备) 内部氢键作用的发生, 将其进行了变温 红外测试(图 9 和图 10). 如图 9 所示, 随温度升高至 $130{ }^{\circ} \mathrm{C}$ 时, $1720 \mathrm{~cm}^{-1}$ 和 $1739 \mathrm{~cm}^{-1}$ 峰位几乎没有变化, 而识别后 thymine 环上 $\mathrm{C}_{4}=\mathrm{O}$ 的新峰 $1671 \mathrm{~cm}^{-1}$ 逐渐向 高波数回移, 并最终稳定在识别前的 $1677 \mathrm{~cm}^{-1}$ 处. 图 10 中, 识别后的新峰 $3398 \mathrm{~cm}^{-1}$ 和 $3210 \mathrm{~cm}^{-1}$ 高温时逐 渐向低波数回移至与识别前峰位相近的 $3319 \mathrm{~cm}^{-1}$ 和 $3203 \mathrm{~cm}^{-1}$ 处(参考图 8). 说明常温下 PDCAI 中腺嘌呤上 $-\mathrm{NH}_{2}$ 和 thymine 环上 $\mathrm{C}_{4}=\mathrm{O}$ 间形成的氢键在高温下逐渐 断裂至完全, PDCAI 和 thymine 各自回归到识别前的状 态.

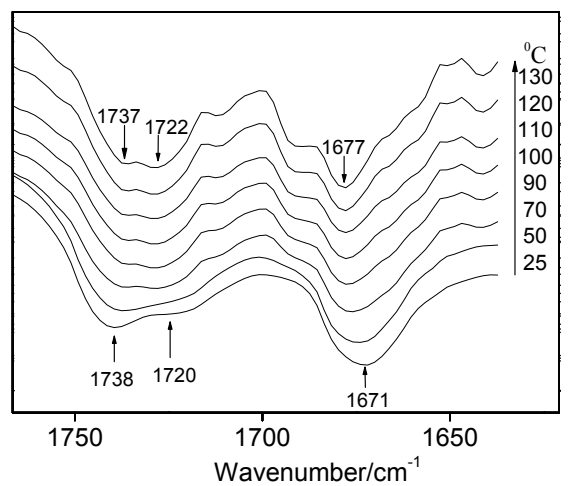

图 $925 \sim 130{ }^{\circ} \mathrm{C}$ 范围内硝酸钾水溶液中制备的 PDCAI/thymine 纳米 聚集体在 $1800 \sim 1520 \mathrm{~cm}^{-1}$ 范围内的变温红外谱图

Figure 9 Variable temperature FT-IR spectra of PDCAI/thymine nano-aggregates prepared from potassium nitrate aqueous solution in $1800 \sim 1520 \mathrm{~cm}^{-1}$ at $25 \sim 130{ }^{\circ} \mathrm{C}$

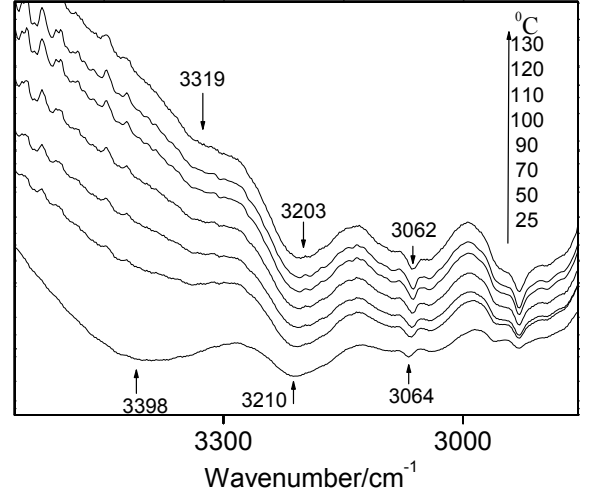

图 $1025 \sim 130{ }^{\circ} \mathrm{C}$ 范围内硝酸钾水溶液中制备的 PDCAI/thymine 纳 米聚集体在 $3500 \sim 2800 \mathrm{~cm}^{-1}$ 范围内的变温红外谱图

Figure 10 Variable temperature FT-IR spectra of PDCAI/thymine nano-aggregates prepared from potassium nitrate aqueous solution in $3500 \sim 2800 \mathrm{~cm}^{-1}$ at $25 \sim 130{ }^{\circ} \mathrm{C}$

\section{3 结论}

模拟 DNA 化学结构, 设计并合成了双亲共聚物聚 (2,2'-(1,10-二氮杂-[18]冠-6-1,10-二基)二乙基 5-((腺嘌 呤-9-基)甲基)间苯二甲酸酯) (PDCAI). SEM 观测到其在 水溶液中自发聚集成宽 40 50 nm 的条带状聚集体，利 用 $\mathrm{K}^{+}$可调控其在水溶液中的聚集形态和与底物 thymine 的氢键识别: 调控后聚集形态转变成棒状、纳米管状或 螺旋棒状; 在水溶液中氢键识别基为 thymine 环上 $\mathrm{C}_{2}=$ $\mathrm{O}$, 而在 $\mathrm{KNO}_{3}$ 水溶液中, 氢键识别发生在 thymine 环上 $\mathrm{C}_{4}=\mathrm{O}$ 位, 说明由于 PDCAI 聚集形态的转变导致底物 thymine 在与其氢键识别过程中进行了识别构象的重组 织, 该研究结果一方面可为研究和揭示聚合物形成螺旋 的分子特征提供参考, 为制备螺旋型聚合物提够一条可 能途径, 另一方面, 该共聚物可在水环境中进行分子自 组装，对互补碱基药物小分子进行包裹、识别和缓释， 可能成为新型仿核酸药物载体.

\section{4 实验部分}

\section{1 实验方法}

共聚物 PDCAI 的结构设计及合成路线见图 11 和图 12.

\section{1 .1 催化剂 $\mathbf{1}$ 的制备}

将 $0.28 \mathrm{~g}$ 无水硫酸镁加入 $20 \mathrm{~mL}$ 无水甲醇中，回流 $2 \mathrm{~h}$, 冷却至室温, 加入 $1.1 \mathrm{~mL}$ 钛酸四丁酯, 搅拌均匀, 备用.

4.1.2 化合物 $\mathbf{5}$ 的合成

将化合物 $4^{[23]} 2.2 \mathrm{~g}$ 溶于 $10 \mathrm{~mL}$ 甲醇中，冰浴冷却至 $0{ }^{\circ} \mathrm{C}$, 加入 $2 \mathrm{~mL}$ 环氧乙烷 $\left(0{ }^{\circ} \mathrm{C}\right)$, 冷凝机制冷回流 $2 \mathrm{~h}$, $2 \mathrm{~h}$ 后撤去冷凝机, 再用普通冷凝水回流 $1 \mathrm{~h}$. 旋干甲醇, 真空减压蒸馏得液体浅黄色产物 5 . 产率 $40 \%,{ }^{1} \mathrm{H}$ NMR (500 MHz, DMSO) $\delta: 3.40 \sim 3.49(\mathrm{~m}, 22 \mathrm{H}), 2.67$ (t, $J=10$ 
$\mathrm{Hz}, 8 \mathrm{H}), 2.52(\mathrm{t}, J=10 \mathrm{~Hz}, 4 \mathrm{H})$.

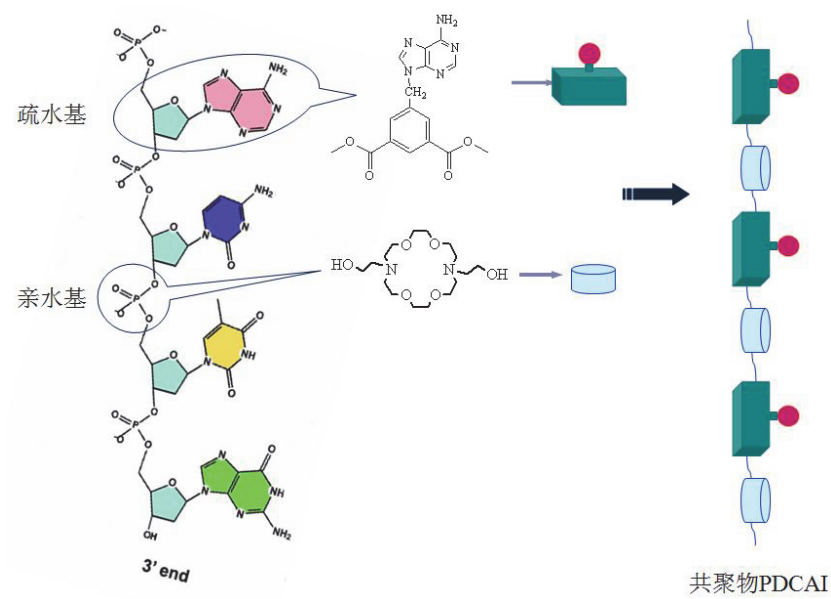

图 11 聚(2,2'-(1,10-二氮杂-[18]冠-6-1,10-二基)二乙基 5-((腺嘌呤-9基)甲基)间苯二甲酸酯) (PDCAI)的结构设计

Figure 11 Structural design of poly(2,2'-(1,10-diaza-[18]crown-61,10-diyl)diethyl 5-((adenin-9-yl)methyl)isophthalate) (PDCAI)
4.1.3 化合物 8 的合成

$\mathrm{N}_{2}$ 保护下, 将化合物 $7^{[23]}(1.72 \mathrm{~g}, 6 \mathrm{mmol})$, 腺嘌呤 $(0.68 \mathrm{~g}, 5 \mathrm{mmol})$, 无水碳酸钾 $(1.4 \mathrm{~g}, 10 \mathrm{mmol})$ 加入到 80 $\mathrm{mL}$ 乙腈中, 加热至回流, $4 \mathrm{~h}$ 后恢复室温, 过滤, 旋干滤 液, 柱层析 $[V($ 氯仿 $): V$ (甲醇 $)=80 ： 1$ ]提纯得白色固体 产物 $0.93 \mathrm{~g}$, 产率: $54.6 \%$ m.p.: $255 \sim 257{ }^{\circ} \mathrm{C},{ }^{1} \mathrm{H}$ NMR $\left(500 \mathrm{MHz}, \mathrm{CDCl}_{3}\right) \delta: 8.64(\mathrm{~s}, 1 \mathrm{H}), 8.40(\mathrm{~s}, 1 \mathrm{H}), 8.17(\mathrm{~s}$, 2H), $7.82(\mathrm{~s}, 1 \mathrm{H}), 5.47(\mathrm{~s}, 2 \mathrm{H}), 3.93(\mathrm{~s}, 6 \mathrm{H})$. Anal. calcd for $\mathrm{C}_{16} \mathrm{H}_{15} \mathrm{~N}_{5} \mathrm{O}_{4}$ : C 56.31, H 4.43, N 20.52; found $\mathrm{C} 55.89, \mathrm{H}$ $4.44, \mathrm{~N} 20.35$.

\subsection{4 聚合物 9 的合成}

将化合物 5 (0.35 g, $1 \mathrm{mmol})$, 化合物 8 (0.35 g, 1 $\mathrm{mmol})$, 抗氧剂 $1010(w=0.22 \%)$ 加入到圆底烧瓶中, $\mathrm{N}_{2}$ 保护下, 油浴加热至 $120{ }^{\circ} \mathrm{C}$, 保持 $1 \mathrm{~h}$ 后冷却至室温, 向该混合物中加入催化剂 $1(w=0.52 \%)$, 逐步升温至 $180{ }^{\circ} \mathrm{C}$, 关闭 $\mathrm{N}_{2}$ ，抽真空，搅拌 $3 \mathrm{~h}$ 后升温至 $200{ }^{\circ} \mathrm{C}$, 再继续反应 $1 \mathrm{~h}$, 停止抽真空, $\mathrm{N}_{2}$ 保护下冷却至室温, 得

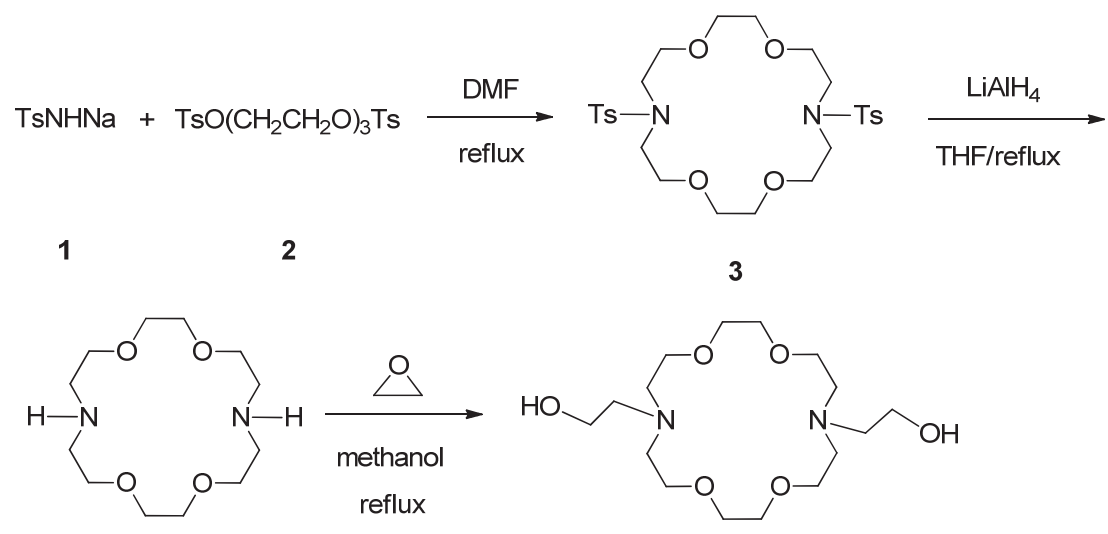

4<smiles>COC(=O)c1cc(Cn2cnc3c(N)ncnc32)cc(C(=O)OC)c1</smiles>

6

5<smiles>COC(=O)c1cc(CBr)cc(C(=O)OC)c1</smiles>

7

8<smiles>COC(C)(C)C(=O)c1cc(Cn2cnc3c(N)ncnc32)cc(C(=O)OCCCN2CCOCCOCCN(CCOC(C)(C)C)CCOCC2)c1</smiles>

9

图 12 聚(2,2'-(1,10-二氮杂-[18]冠-6-1,10-二基)二乙基 5-((腺嘌呤-9-基)甲基)间苯二甲酸酯) (PDCAI)的合成路线

Figure 12 Synthetic routes of poly(2,2'-(1,10-diaza-[18]crown-6-1,10-diyl)diethyl 5-((adenin-9-yl)methyl)isophthalate) (PDCAI, 9) 
到褐色粗产物. 多次用氯仿洗粗产物得到褐色固状聚合 物 9. ${ }^{1} \mathrm{H}$ NMR (500 MHz, DMSO) $\delta: 8.39 \sim 8.06(\mathrm{~m}, 5 \mathrm{H})$, $7.27(\mathrm{~s}, 2 \mathrm{H}), 5.50(\mathrm{~s}, 2 \mathrm{H}), 4.39(\mathrm{~s}, 2 \mathrm{H}), 3.28 \sim 3.54(\mathrm{bs}$, methylene protons next to $\mathrm{O}$ of diaza-crown), $2.79 \sim 2.68$ (bs, methylene protons next to $\mathrm{N}$ of diaza-crown) SEC-MALLS: $M_{\mathrm{n}}=3.384 \mathrm{e}^{+04}, M_{\mathrm{w}}=4.534 \mathrm{e}^{+04}, M_{\mathrm{w}} / M_{\mathrm{n}}=$ 1.34 .

\subsubsection{PDCAI、PDCAI/thymine 聚集体的制备}

在 $50 \mathrm{~mL}$ 磨口瓶内, 将 PDCAI 或 PDCAI/thymine (官能团摩尔比 $1 ： 1$ ) 的混合物溶于 DMF, 真空减压抽除 $\mathrm{DMF}$, 使共聚物在容器壁上形成一层薄膜, 加入去离子 水或 $\mathrm{KNO}_{3}$ 水溶液定容, 使 PDCAI 浓度为 $1 \times 10^{-4}$ $\mathrm{mol} / \mathrm{L}$, 超声 $4 \mathrm{~h}$, 冰水浴冷却 $0.5 \mathrm{~h}$, 自然恢复室温, 将 制备的聚集体水溶液直接滴到硅片上, 真空干燥, 待测 扫描电镜 ${ }^{[15]}$. 将该溶液冷冻干燥, 则 PDCAI 或 $\mathrm{PDCAI} /$ thymine 聚集体将保持在水或 $\mathrm{KNO}_{3}$ 水溶液中的 聚集状态, 待测红外.

\section{References}

[1] Ben-Naim, A. Hydrophobic Interactions, Plenum Press, New York, 1980, p. 311.

[2] Saenger, W. Principles of Nucleic Acid Structure, Springer, New York, 1984, p. 298.
[3] Gao, Y. X.; Hu, J.; Ju, Y. Acta Chim. Sinica 2016, 74(4), 312. (高玉 霞, 胡君, 巨勇, 化学学报, 2016, 74(4), 312.)

[4] Liu, C.; Yu, G.; Huang, C. Y.; Wang, C. S. Acta Chim. Sinica 2015 , 73(4), 357. (刘畅, 于歌, 黄翠英, 王长生, 化学学报, 2015, 73(4), 357.)

[5] Hawker, C. J.; Wooley, K. L. Science 2005, 309, 1200.

[6] Berti, D.; Luisi, P. L.; Baglioni, P. Colloids \& Surfaces 2000, 167, 95

[7] Eschenmoser, A. Science 1999, 284, 2118

[8] Zhang, L.; Peritz, A.; Meggers, E. J. Am. Chem. Soc. 2005, 127, 4174.

[9] Yoshimoto, K.; Nishizawa, S.; Minagawa, M. J. Am. Chem. Soc. 2003, 125, 8982.

[10] Nowick, J. S.; Cao, T.; Noronha, G. J. Am. Chem. Soc. 1994, 116, 3285 .

[11] Baglioni, P.; Berti, D. Colloid Interface Sci. 2003, 8, 55.

[12] Miao, W.; Du, X.; Liang, Y. Langmuir 2003, 19, 5389.

[13] Yashima, E.; Maeda, K. Chem.-Eur. J. 2004, 10, 42.

[14] Yang, M. J. Beijing Med. Univ. 1998, 30(2), 97. (杨铭，北京医科大 学学报, 1998, 30(2), 97.)

[15] Pedersen, C. J. J. Am. Chem. Soc. 1967, 89, 7017.

[16] Cram, D. J. Angew. Chem., Int. Ed. Engl. 1988, 27, 1009.

[17] Huang, F. H.; Ji, X. F.; Yao, Y. J. Am. Chem. Soc. 2013, 135, 74.

[18] Ji, X.; Dong, S.; Wei, P.; Xia, D.; Huang, F. Adv. Mater. 2013, 25, 5725 .

[19] Masuda, M.; Okada, Y. Macromolecules 2000, 33, 9233.

[20] Buchet, R.; Sandorfy, C. J. Phys. Chem. 1983, 87, 275.

[21] Alabugin, I. V.; Manoharan, M.; Peabody, S.; Weinhold, F. J. Am. Chem. Soc. 2003, 125, 5973.

[22] Florian, J. Spectrochim. Acta 1993, 49A, 921.

[23] Zhang, Y. J.; Xie, B. Acta Chim. Sinica 2012, 70, 499. (张㻦健, 谢 涁, 化学学报, 2012, 70, 499.)

(Cheng, B.) 